\title{
The surplus value of Azorean Camellia sinensis flowers as an important contributor affecting the nutraceutical benefits of green tea quality
}

\begin{abstract}
Leaves from Camellia sinensis have been used to make teas for a long time, however, less attention has been paid to the flowers, which is a waste of an abundant resource that should be valorized. This study evaluates the representative tea metabolites content (dry weight basis) and antioxidant properties of Azorean $C$. sinensis flowers as compared to commercial Gorreana green tea (GorGT), the only one produced in Europe. We determined the variability of catechins, caffeine and L-theanine (THEA) by RP-HPLC/DAD and the confirmation of THEA by GC/MS. The antioxidants activities were determined by FRSA, FRAP and FIC methods. The total phenolics and total flavonoids were determined by the Folin-Ciocalteu and colorimetric methodologies. The results showed that flowers presented higher L-theanine $(5.88 \mathrm{mg} / \mathrm{g})$ but lower caffeine $(4.10 \mathrm{mg} / \mathrm{g})$ contents than GorGT (2.59 and $12.42 \mathrm{mg} / \mathrm{g}$, respectively) and, therefore, a lower caffeine/theanine ratio ( 0.70 versus 4.80). The esterified catechins content (in percentage of total catechins) showed similar values for flowers $(85.34 \%)$ and GorGT $(86.45 \%)$, and the individual catechins decreased as follows: epigallocatechin-3-gallate > epicatechin-3-gallate $\gg$ gallocatecin-3-gallate. In antioxidant assays, GorGT presented better FRSA and FRAP but similar FIC as compared to flowers. Also, the total phenolics and flavonoids contents of dried extract presented higher values in GorGT: $321.56 \mathrm{mg}$ gallic acid equivalents (GAE)/g and $47.17 \mathrm{mg}$ rutin equivalents (RE)/g as compared to $141.94 \mathrm{mg} \mathrm{GAE} / \mathrm{g}$ and $24.14 \mathrm{mg} \mathrm{RE} / \mathrm{g}$, respectively, for flowers. This study represents the first contribute to Azorean C. sinensis flowers valorization as a new-added material with nutraceutical benefits.
\end{abstract}

Keywords: $C$. sinensis waste valorization, Tea (C. sinensis) flower, commercial green tea, caffeine/L-theanine ratio, catechin profiles, total phenolics and flavonoids, antioxidant activities, cognitive functions, human health, RP-HPLC analysis
Volume 7 Issue 6 - 2019

\author{
Lisete Paiva, ${ }^{1,2}$ Elisabete Lima, ${ }^{2,3}$ Madalena \\ Motta,' José Baptista ${ }^{2,3}$ \\ 'Gorreana Tea Plantation, Gorreana, Portuga/ \\ ${ }^{2}$ Department of Physics, Chemistry and Engineering (DPCE) and \\ Biotechnology Centre of Azores (CBA), University of Azores, \\ Portugal \\ ${ }^{3}$ Institute of Agricultural and Environmental Research and \\ Technology (IITAA), University of Azores, Portugal
}

\begin{abstract}
Correspondence: José Baptista, Department of Physics, Chemistry and Engineering (DPCE) and Biotechnology Centre of Azores (CBA), University of Azores, 9500-32I Ponta Delgada, São Miguel,Azores, Portugal, Tel +35 I-296-650-182, Email jose.ab.baptist@uac.pt
\end{abstract}

Received: December 03, 2019 | Published: December 23,
2019
Abbreviations: CAF, caffeine; DW, dry weight; ECDs, epicatechin derivatives; GAE, gallic acid equivalents; GorGT, commercial Gorreana green tea; THEA, L-theanine; RE, rutin equivalents; TP, tea polyphenols

\section{Introduction}

Tea (Camellia sinensis L.) is one of the most ancient and the most popularly consumed non-alcoholic beverage worldwide, after water Tea plant, originally from Southeast China, gradually expanded into many tropical and subtropical countries. Since the last decade of the $19^{\text {th }}$ century, tea is also produced in one unique place in Europe, the volcanic São Miguel Island of the Azores Archipelago (Portugal) that is characterized by an oceanic climate, with mild temperature all year around. ${ }^{1}$ It is well established that the chemical composition of tea, and therefore tea quality, is significantly influenced by many factors such as: the region of tea production, plant variety, growing environment, plucking season, age leaf and processing conditions. ${ }^{2}$

In recent years, a remarkable growing body of research describes many beneficial effects of tea intake (e.g. anticancer, antidiabetic, antimicrobial, anti-obesity, antioxidant, bone density, cardioprotective, cognitive function and neuropharmacological potential) being some of them particularly relevant to senior adults..$^{3-7}$ Tea is a major source of dietary flavonoids, particularly the catechins, that are the main contributors to the health-promoting effects of tea, as demonstrated by numerous in vitro and in vivo studies. It is also known that epigallocatechin-3-gallate, a major polyphenol in green tea, has been the focus of research owing to it multiple protective effects against chronic diseases. ${ }^{4,8}$ However, further research is warranted on the potential for synergy effects among the tea bioactive flavonoids, caffeine (CAF) and L-theanine (THEA), a non-protein amino acid "unique" to tea that accounting for up to $50 \%$ of all free amino acids. ${ }^{3,9}$ Recent studies reports that THEA (5- $N$-ethyl glutamine) has many biological and physiological activities such as: calming effect on the mental state by lowering blood pressure; inhibits CAF's side effects; stimulates the release of dopamine, a brain's neurotransmitter responsible for confidence and sense of well-being; stimulates the production of alfa brain waves (an electromagnetic oscillation in the frequency range of $8-13 \mathrm{~Hz}$ ) in the occipital and parietal regions of human brain, creating, consequently, a state of deep relaxation and mental alertness; enhances anti-tumor activity and promotes neuroprotection. ${ }^{9-11}$

Besides catechins, the THEA and CAF are pharmacologically important tea constituents, especially due to their antagonist effects on the central nervous system. ${ }^{12,13}$ So, the quantitative analysis of these compounds, and consequently their ratio, is essential to assess the degree of the stimulating effect of tea drinks, and it can be stated that tea samples with lower CAF/THEA ratio have less stimulating effect. ${ }^{12}$ On the other hand, while the phytochemical composition 
and beneficial health properties of tea leaves have been extensively studied, less attention has been given to those of flowers of $C$. sinensis plant, which are a wasted and abundant resource that may have wide applications in near future. ${ }^{14}$

Knowing that metabolites are not limited to a sole plant tissue and can be distributed among several tissues, one question remains to be answered: What is the contribution of flowers to the Azorean (Gorreana) green tea quality? To answer this question, the determination of important tea quality markers (catechins, total phenolics, total flavonoids, CAF and THEA contents, as well as antioxidant activities, namely, free radical scavenging activity, ferric reducing antioxidant power and ferrous ion-chelating activity) in tea plant flowers and in commercial Gorreana green tea (GorGT) samples were performed in order to investigate a new alternative drinking beverage from tea leaves plus tea flowers with diverse beneficial effects on human health. To the authors' knowledge, this type of commercial beverage has never been made.

\section{Materials and methods}

\section{Chemicals and reagents}

THEA (lot 606121) was generously donated by Taiyo Kagaku International Inc. (Boise, ID). Catechins, namely, catechin (C), epicatechin (EC), gallocatechin (GC), epigallocatechin (EGC), gallocatechin-3-gallate (GCG), epigallocatechin-3-gallate (EGCG) and epicatechin-3-gallate (ECG), CAF, gallic acid, rutin, 2,2-diphenyl-1-picrylhydrazyl (DPPH), butylated hydroxytoluene (BHT), ethylenediaminetetraacetic disodium salt (EDTA), FolinCiocalteu reagent, potassium ferricyanide, iron (II) chloride, iron (III) chloride, aluminum chloride, ferrozine and $o$-phthaldialdehyde (OPA) were obtained from Sigma-Aldrich (St. Louis, MO, USA). $\beta$-mercaptoethanol, potassium monobasic phosphate, sodium borate, sodium acetate, sodium carbonate, potassium acetate, sodium phosphate and orthophosphoric acid were purchased from E. Merck (Darmstad, Hessen, Germany). Acetonitrile, tetrahydrofuran, methanol, chloroform and ethyl acetate, HPLC-grade, were obtained from Riedel-de Häen (Aktiengesellschaft, Seelze, Germany) and other chemicals and solvents were analytical grade. Deionised water was obtained from an in-house Milli-Q water purification system (Millipore, Bedford, MA, USA).

\section{Samples collection, preparation and extraction}

Samples of GorGT and flowers of $C$. sinensis were provided by Gorreana Tea Plantation (São Miguel, Azores). The freshly plucked flower samples were indoor-withered at $25-30^{\circ} \mathrm{C}$ to achieve a relative humidity of $70 \%$ and then dried at $40^{\circ} \mathrm{C}$ until constant weight. The protocol for the extraction of total tea polyphenols (TP) and CAF from the samples was a slightly modified methodology described by Baptista et al., ${ }^{1}$ and THEA extraction was performed according to Baptista et al., ${ }^{10}$ protocol.

\section{RP-HPLC analysis of catechins and CAF}

The variability of the catechin profiles and CAF content in the samples under study was determined by RP-HPLC/DAD because it provides an efficient and quick analysis method for these tea compounds, as validated in previous research on tea extracts. ${ }^{1}$ The analysis was carried out on an Ultropac Spherisorb ODS2 column (100 x $4.6 \mathrm{~mm}$ id) from LKB (Bromma, Sweden). The baseline separation was achieved with a binary elution as follows: $100 \%$ A (acetonitrile:ethyl acetate: $0.1 \%$ orthophophoric acid:water, 4.25:1:44.75:50, $\mathrm{v} / \mathrm{v} / \mathrm{v}$ ) for $10 \mathrm{~min}$, followed by a linear gradient between the phase A and phase B (acetonitrile:water, 1:1, v/v), reaching $20 \% \mathrm{~B}$ in $10 \mathrm{~min}$ and held at this composition until the end of the run, at a flow rate of $0.8 \mathrm{~mL} / \mathrm{min}$ and detection wavelength of $280 \mathrm{~nm}$. The individual catechins contents were expressed as a percentage by mass of the total catechins on a dry weight (DW) basis, and the total catechins, total epicatechin derivatives (ECDs) and total esterified catechins were obtained by summation as follows: GC + $\mathrm{EGC}+\mathrm{C}+\mathrm{EC}+\mathrm{EGCG}+\mathrm{GCG}+\mathrm{ECG} ; \mathrm{EGC}+\mathrm{EC}+\mathrm{EGCG}+$ $\mathrm{ECG}$ and $\mathrm{EGCG}+\mathrm{GCG}+\mathrm{ECG}$, respectively. The CAF content was expressed in $\mathrm{mg} / \mathrm{g} \mathrm{DW}$.

\section{RP-HPLC and GC/MS analysis of THEA after sample derivatisation}

The THEA determination in the sample extracts was performed by HPLC/DAD analysis on RP-C18 column after derivatisation with OPA, according to the methodology published by Baptista et al., ${ }^{10}$ and THEA content was expressed in $\mathrm{mg} / \mathrm{g}$ DW. The detection by GC/MS at $\mathrm{m} / \mathrm{z} 129,111,87,84,72,56$ was used for the confirmation of THEA pooled peak from the HPLC separation. The conditions of the GC/MS analysis were described by Baptista et al. ${ }^{10}$

\section{DPPH free radical scavenging activity (FRSA) determination on TP extracts}

The FRSA of TP extracts of GorGT and tea flowers on DPPH radical was determined according to the method of Molyneux ${ }^{15}$ with some modifications. ${ }^{16}$ BHT was used as reference sample. The results were expressed as $\mathrm{EC}_{50}$ value $(\mu \mathrm{g} / \mathrm{mL})$, which is defined as the sample concentration that can quench fifty percent of DPPH free radicals. A lower $\mathrm{EC}_{50}$ value means a higher antioxidant activity.

\section{Ferric reducing antioxidant power (FRAP) determination on TP extracts}

The FRAP of TP extracts was determined according to the method of Oyaizu ${ }^{17}$ with some modifications, ${ }^{16}$ and was evaluated on the basis of their abilities to reduce $\mathrm{Fe}^{3+}$ complex to $\mathrm{Fe}^{2+}$. BHT was used for comparison. The results were expressed as $\mathrm{EC}_{50}$ value $(\mu \mathrm{g} / \mathrm{mL})$ that is the effective concentration at which the absorbance was 0.5 for reducing power and were obtained by interpolation from linear regression analysis of concentration versus absorbance at $700 \mathrm{~nm}$. A lower $\mathrm{EC}_{50}$ value means a higher antioxidant activity.

\section{Ferrous ion-chelating (FIC) activity determination on TP extracts}

Chelating ability of TP extracts were determined according to the modified method of Wang et al., ${ }^{18}$ with some modifications, ${ }^{16}$ by measuring the percentage inhibition of the $\mathrm{Fe}^{2+}-$ ferrozine complex formation. EDTA was used as reference standard.

\section{Total phenolic content (TPC) determination in TP extracts}

TPC was determined by using Folin-Ciocalteu colorimetric methodology based on the oxidation/reduction reaction as described by Waterhouse ${ }^{19}$ with some modifications. ${ }^{16}$ The gallic acid was used as a standard and the results were expressed as $\mathrm{mg}$ of gallic acid equivalents per gram of dried extract (mg GAE/g DE). 


\section{Total flavonoids content (TFC) determination in TP extracts}

TFC was determined by following colorimetric method of Chang et al. ${ }^{20}$ with some modifications. ${ }^{16}$ TFC was expressed as mg rutin equivalents per gram of dried extract (mg RE/g DE).

\section{Statistical analysis}

All determinations were performed in triplicate and the results were expressed as means \pm standard deviations (SD). One-way analysis of variance test (ANOVA) was carried out to assess for any significant differences between the means. Differences between means at the 5\% $(p<0.05)$ level were considered statistically significant.

\section{Results and discussion}

The determination of THEA, catechins and CAF in flowers and in GorGT samples were assessed by HPLC, and the data are summarized in Table 1. The flowers presented the highest content of THEA (see Figure 1 for THEA separation and Figure 2 for the GC/MS THEA confirmation) with an average value of $5.88 \mathrm{mg} / \mathrm{g}$ DW as compared to $2.59 \mathrm{mg} / \mathrm{g}$ DW for GorGT samples. A similar pattern has been reported by Wang et al., ${ }^{11}$ for Chinese teas and tea plant flowers. However, it should be highlighted that THEA levels in tea is significantly influenced by several factors, such as: growth altitude and season of tea production, climate conditions (e.g. sunlight intensity), horticultural practices, soil properties, age leaf and, particularly, the processing conditions like withering duration, and time and temperature of the drying process. ${ }^{12,21,22}$ On the other hand, the total esterified catechins content (in percentage of the total catechins) showed similar values for flowers $(85.34 \%)$ and GorGT $(86.45 \%)$. Furthermore, in both samples, the individual esterified catechins decreased as follows: EGCG $>$ ECG 》GCG. A similar pattern has been reported for Chinese tea flowers extracts, ${ }^{23}$ and Korean green tea extracts. ${ }^{21}$ Our results also revealed that flowers contain less EGCG and more ECG than GorGT samples. Relatively to the total ECDs content, flowers presented a lower value $(83.18 \%)$ than GorGT $(90.05 \%)$, and the individual ECDs decreased in both samples as follows: EGCG $>$ ECG $\gg$ EC $>$ EGC. Similar results of tea catechins were reported by other authors showing that the total catechins content in tea flowers was lower or, in some cases, comparable with that in tea leaves. ${ }^{14,24}$ It should also be highlighted that the catechins content in tea flowers increase after budding, reaching their maximum values when the petals started to split and then decreased to their values at the full bloom stage. ${ }^{14}$ Concerning the CAF content, our results show that flowers presented the lowest value of $4.10 \mathrm{mg} / \mathrm{g}$ DW as compared with $12.42 \mathrm{mg} / \mathrm{g}$ DW from GorGT that is a contrary pattern relatively to THEA content, therefore, the CAF/THEA ratio was remarkably lower in flowers $(0.70)$ than in GorGT (4.80). Thus, the tea flowers have lower stimulant effect on the human nervous system as compared with GorGT. On the other hand, according to Fujimori \& Ashihara, ${ }^{25} \mathrm{CAF}$ presented in tea flowers is synthesized in the tea flowers and not transported from the tea leaves. Lin et al., ${ }^{24}$ also reported lower levels of CAF in various fresh tea flowers from Taiwan ( 3 to $8 \mathrm{mg} / \mathrm{g}$ ) as compared to fresh tea leaves in various commercial teas ( 23 to $49 \mathrm{mg} / \mathrm{g}$ ). Regarding the CAF/THEA ratio, Boros et al., ${ }^{12}$ reported slightly lower values (1.57 to 4.22$)$ for sixteen commercial green tea analyzed as compared to GorGT (4.80). Despite the practical relevance of the CAF and THEA contents in tea, there are only a few papers dealing with the simultaneous quantification of these metabolites. ${ }^{12}$
Table I Comparison of the contents of individual catechins (in \% of total catechins), caffeine and L-theanine $(\mathrm{mg} / \mathrm{g})$ in tea plant flowers and in commercial Gorreana green tea (GorGT) samples from Azorean Camellia sinensis ${ }^{\mathrm{a}}$

\begin{tabular}{lll}
\hline \multirow{2}{*}{ Compound } & \multicolumn{2}{l}{ Content ${ }^{\mathrm{b}}$ in C. sinensis samples } \\
\cline { 2 - 3 } & Flowers & GorGT \\
\hline GC & $3.43 \pm 0.05$ & $0.48 \pm 0.03$ \\
EGC & $1.31 \pm 0.02$ & $2.41 \pm 0.06$ \\
C & $1.33 \pm 0.06$ & $1.51 \pm 0.05$ \\
EC & $8.61 \pm 0.08$ & $9.16 \pm 0.19$ \\
EGCG & $40.21 \pm 1.66$ & $49.26 \pm 1.84$ \\
GCG & $12.08 \pm 0.13$ & $7.97 \pm 0.21$ \\
ECG & $33.05 \pm 1.22$ & $29.22 \pm 0.85$ \\
Total ECDs & $83.18 \pm 3.13$ & $90.05 \pm 3.39$ \\
Total Est. Cat. & $85.34 \pm 3.52$ & $86.45 \pm 3.53$ \\
CAF & $4.10 \pm 0.21$ & $12.42 \pm 0.26$ \\
THEA & $5.88 \pm 0.08$ & $2.59 \pm 0.04$ \\
CAF/THEA ratio & 0.7 & 4.8
\end{tabular}

aValues are mean $\pm S D(n=3)$. GC, (-)-gallocatechin; EGC, (-)-epigallocatechin; C, (+)-catechin; EC, (-)-epicatechin; EGCG, (-)-epigallocatechin-3-gallate; GCG, (-)-gallocatechin-3-gallate; ECG, (-)-epicatechin-3-gallate; Total ECDs (epicatechin derivatives), sum of EGC, EC, EGCG and ECG; Total Est. Cat. (esterified catechins), sum of EGCG, GCG and ECG; CAF, caffeine; THEA, L-theanine. ${ }^{\circ}$ Dry weight basis

The antioxidant properties, TPC and TFC results of the studied TP extracts are illustrated in Table 2. The flowers presented the FRSA and FRAP EC50 values of 17.00 and $17.70 \mu \mathrm{g} / \mathrm{mL}$, as compared to 5.61 and $6.81 \mu \mathrm{g} / \mathrm{mL}$ for GorGT, respectively, revealing higher antioxidant activity in GorGT than in flowers, however, the FRSA of flowers was better than that in BHT $\left(\mathrm{EC}_{50}\right.$ value of $\left.20.80 \mu \mathrm{g} / \mathrm{mL}\right)$ used, in the same conditions, as a positive control. The results also revealed that FRSA and FRAP values followed a similar pattern in the samples under study, which is explained by the fact that both assays rely on an electron/hydrogen donation mechanism. Yang et al., ${ }^{26}$ reported a significantly lower FRSA (EC50 value of $47.6 \mu \mathrm{g} / \mathrm{mL}$ ) for Chinese tea flowers extracts as compared to Azorean tea flowers extracts, and Chan et al., ${ }^{27}$ reported a lower FRSA $\left(\mathrm{EC}_{50}\right.$ value of $\left.13 \mu \mathrm{g} / \mathrm{mL}\right)$ for commercial Sea Dyke green tea as compared with GorGT. Moreover, Lin et al., ${ }^{24}$ reported that both tea flower and tea leaf extracts exhibit strong hydroxyl radical-scavenging effects in the Fenton reaction system and nitric oxide suppressing effects in LPS-induced RAW 264.7 cells. However, it should be pointed out that the study on antioxidant activity of tea flowers has been rarely reported, in contrary to the antioxidant activity of tea that has been studied extensively. Since metal chelating capacity is claimed as one of the important mechanisms of antioxidant activity, ${ }^{18}$ FIC assay was also chosen to better characterize the antioxidant activity of tea and flowers extracts. As shown in Table 2, flowers and GorGT presented a similar value (46.15\% and $45.12 \%$, respectively) that, as compared to the synthetic antioxidant EDTA, a potent metal-ion chelator used in this study as a positive control, is considered a moderate FIC activity. Similar 
results were reported by previous studies who show that the ECDs contents, particularly the higher levels of EGCG and ECG, were the key factor affecting the antioxidant activity of tea extracts in FRAP and DPPH assays, and that the catechins also have metal chelation potential..$^{21,23,26}$ Concerning the TPC and TFC, the GorGT presented the highest contents, showing the values of $321.56 \mathrm{mg} \mathrm{GAE} / \mathrm{g}$ and $47.17 \mathrm{mg} \mathrm{RE} / \mathrm{g}$ as compared to $141.94 \mathrm{mg} \mathrm{GAE} / \mathrm{g}$ and $24.14 \mathrm{mg} \mathrm{RE} / \mathrm{g}$ for flowers, respectively, which is well correlated with the higher FRSA and FRAP exhibited by GorGT samples. Our results were higher than those reported by Nibir et al. ${ }^{28}$ in different tea varieties of Bangladesh. However, it should be noted that comparison of data between different studies is very difficult when different raw material, processing conditions, extraction protocol, analytical methods, and units were used, among other factors.

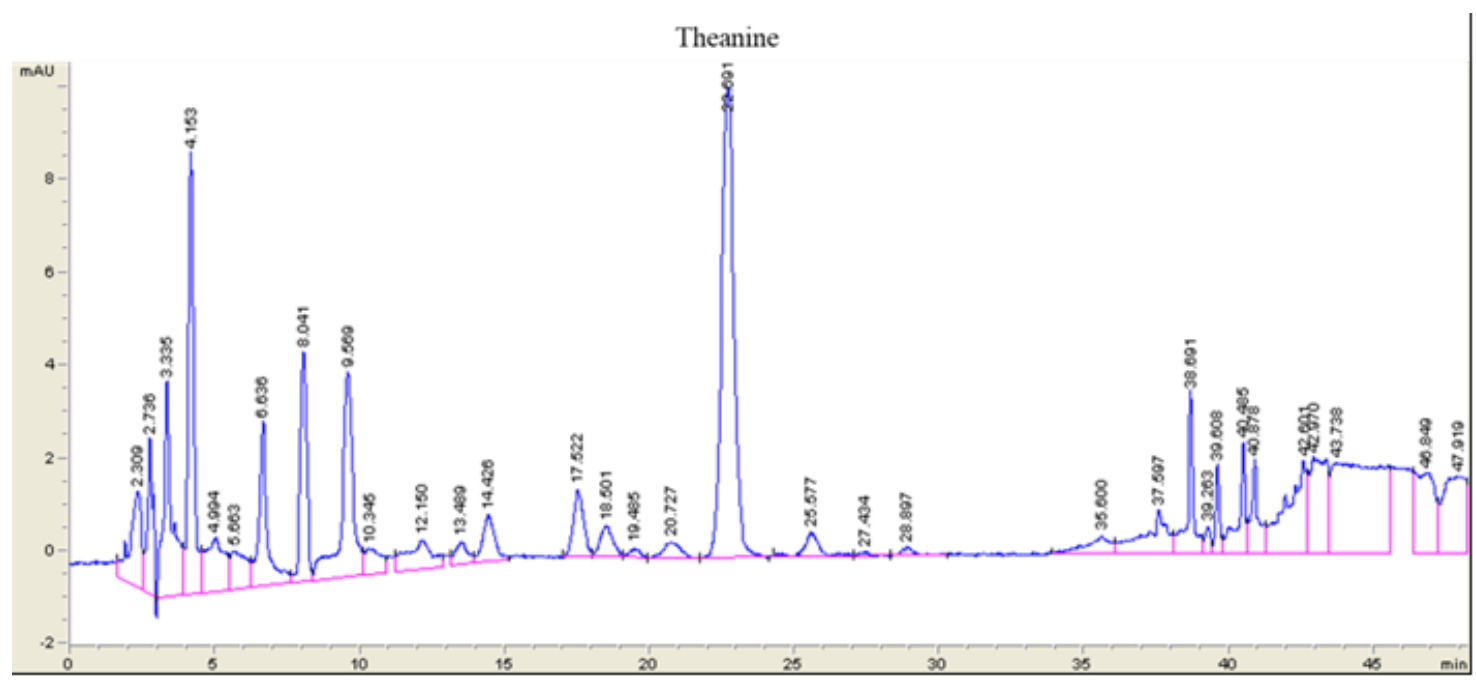

Figure I L-theanine determination by RP-HPLC/DAD of Azorean tea plant flowers. Analytical conditions were described by Baptista et al.'

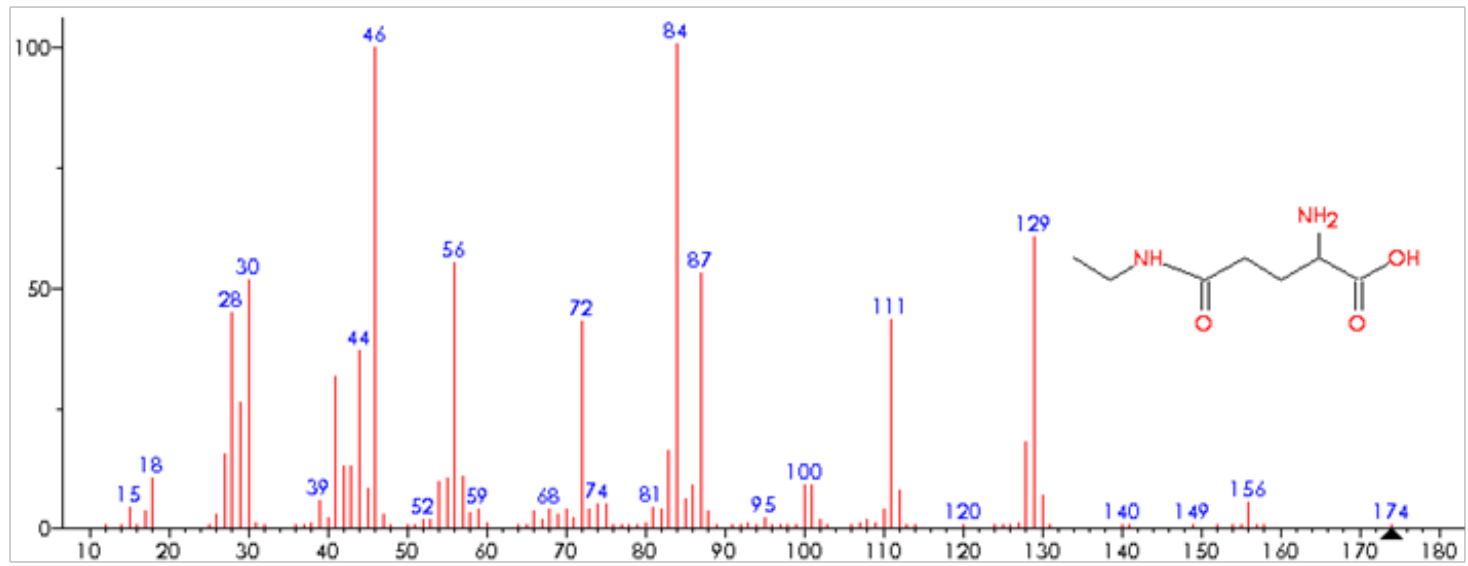

Figure 2 L-theanine confirmation by GC/MS. Analytical conditions of the pooled peak from HPLC separation were described by Baptista et al.'

Table 2 Free radical scavenging activity (FRSA), ferric reducing antioxidant power (FRAP), ferrous ion-chelating (FIC) activity, and contents of total phenolics (TPC) and total flavonoids (TFC) in dry extracts (DE) of tea plant flowers and commercial Gorreana green tea (GorGT) samples from Azorean Camellia sinensis

\begin{tabular}{llllll}
\hline $\begin{array}{l}\text { C. sinensis samples and } \\
\text { control }\end{array}$ & $\begin{array}{l}\text { FRSA }\left(E_{50}{ }^{b}, \mu g /\right. \\
\mathbf{m L})\end{array}$ & $\begin{array}{l}\text { FRAP }\left(E_{50}{ }^{c}, \mu g /\right. \\
\mathbf{m L})\end{array}$ & FIC (\%) & $\begin{array}{l}\text { TPC (mg GAE/g } \\
\text { DE) }\end{array}$ & $\begin{array}{l}\text { TFC (mg RE/g } \\
\text { DE) }\end{array}$ \\
\hline Flowers & $17.00 \pm 0.91$ & $17.70 \pm 0.18$ & $46.15 \pm 2.02$ & $141.94 \pm 2.48$ & $24.14 \pm 0.82$ \\
GorGT & $5.61 \pm 0.67$ & $6.81 \pm 0.16$ & $45.12 \pm 2.08$ & $321.56 \pm 3.15$ & $47.17 \pm 1.96$ \\
BHT & $20.80 \pm 0.60$ & $5.60 \pm 0.09$ & - & - & - \\
EDTA & - & - & $97.64 \pm 0.58$ & - & -
\end{tabular}

${ }^{a}$ Values are mean $\pm S D(n=3)$. GAE, gallic acid equivalentes; RE, rutin equivalentes; BHT, butylated hydroxytoluene; EDTA, ethylenediaminetetraacetic disodium

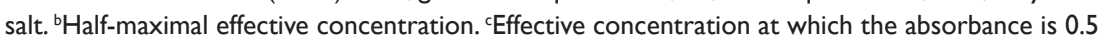


Overall, results from the present research indicate that a new alternative drinking beverage from tea leaves plus tea flowers with less CAF and more THEA contents may offer an additional health benefits for people with lower CAF metabolism and/or senior individuals as compared to green tea itself.

\section{Conclusion}

This comparative study provides, for the first time, an evaluation of the tea functional molecules content and antioxidant properties of Azorean $C$. sinensis flowers in order to investigate a new alternative beverage from tea leaves plus tea flowers with diverse beneficial effects on human health. Compared to Azorean GorGT, the tea flowers have equivalent catechins profiles, which imply that tea flowers may exhibit similar beneficial health properties. In fact, it is well known that catechins group is the main contributor to the healthpromoting effects of green tea. In addition, the tea flowers presented more THEA and less CAF and, therefore, a lower CAF/THEA ratio, which imply that tea flowers have lower stimulant effect on the human nervous system as compared with GorGT. Furthermore, tea flowers have similar FIC activity but lower FRSA, FRAP, TPC and TFC as compared with GorGT, however, the FRSA of flowers was better than that of the synthetic commercial antioxidant BHT. The results suggest that from an economic point of view, both tea leaves and tea flowers should be treated equally, which would greatly reduce the cost of commercial green tea by the addition of one natural value-added product. However, additional research is needed in order to find the right percentage of flowers to produce the finest blend of this novel green tea that maximize the beneficial health-related properties on human health.

\section{Author contributions}

J.B. wrote the manuscript. L.P., E.L. and M.M. contributed to the tea flower-related research aspects carried out by the authors. All authors reviewed the manuscript.

\section{Conflicts of interest}

The authors declare no conflict of interest.

\section{References}

1. Baptista J, Lima E, Paiva L, et al. Value of off-season fresh Camellia sinensis leaves. Antiradical activity, total phenolics content and catechin profiles. Food Sci Technol. 2014;59(2):1152-1158.

2. Tounekti T, Joubert E, Hernández I, et al. Improving the polyphenol content of tea. Crit Rev Plant Sci. 2013;32(3):192-215.

3. Bolling BW, Chen CY, Blumberg JB. Tea and health: preventive and therapeutic usefulness in the elderly? Curr Opin Clin Nutr Metab Care. 2009;12(1):42-48.

4. Chacko SM, Thambi PT, Kuttan R, et al. Beneficial effects of green tea: a literature review. Chin Med. 2010;5:13-14.

5. Khan N, Mukhtar H. Tea and health: studies in humans. Curr Pharm Des. 2013;19(34):6141-6147.

6. Rubab S, Rizwani GH, Bahadur S, et al. Neuropharmacological potential of various morphological parts of Camellia sinensis L. Saudi J Biol Sci. 2020;27(1):567-573.

7. Xu Q, Langley M, Kanthasamy AG, et al. Epigallocatechin gallate has a neurorescue effect in a mouse model of Parkinson disease. $J$ Nutr. 2017;147(10):1926-1931.

8. Khan N, Mukhtar H. Tea polyphenols in promotion of human health Nutrients. 2019;11(1):39.

9. Türközü D, Şanlier N. L-theanine, unique amino acid of tea, and its metabolism, health effects, and safety. Crit Rev Food Sci Nutr. 2017;57(8):1681-1687.

10. Baptista J, Lima E, Paiva L, et al. Comparison of Azorean tea theanine to teas from other origins by HPLC/DAD/FD. Effect of fermentation, drying temperature, drying time and shoot maturity. Food Chem. 2012;132(4):2181-2187.

11. Wang L, Xu R, Hu B, et al. Analysis of free amino acids in Chinese teas and flower of tea plant by high performance liquid chromatography combined with solid-phase extraction. Food Chem. 2010;123(4):12591266.

12. Boros K, Jedlinszki N, Csupor D. Theanine and caffeine content of infusions prepared from commercial tea samples. Pharmacogn Mag. 2016;12(45):75-79.

13. Nehlig A, Daval JL, Debry G. Caffeine and the central nervous system: mechanisms of action, biochemical, metabolic and psychostimulant effects. Brain Res Rev. 1992;17(2):139-170.

14. Chen Y, Zhou Y, Zeng L, et al. Occurrence of functional molecules in the flowers of tea (Camellia sinensis) plants: evidence for a second resource. Molecules. 2018;23(4):790.

15. Molyneux P. The use of the stable free radical diphenylpicrylhydrazyl (DPPH) for estimating antioxidant activity. Songklanakarin J Sci Technol. 2004;26(2):211-219.

16. Paiva L, Lima E, Neto AI, et al. Seasonal variability of the biochemical composition and antioxidant properties of Fucus spiralis at two Azorean Islands. Mar Drugs. 2018;16(8):248

17. Oyaizu M. Studies on products of browning reactions: antioxidative activities of products of browning reaction prepared from glucosamine. Jpn J Nutr. 1986;44(6):307-315.

18. Wang T, Jónsdóttir R, Ólafsdóttir G. Total phenolic compounds, radical scavenging and metal chelation of extracts from Icelandic seaweeds. Food Chem. 2009;116(1):240-248.

19. Waterhouse AL. Determination of total phenolics. In R.E. Wrolstad (Ed.), Current Protocols in Food Analytical Chemistry. New York, NY, USA: John Wiley \& Sons; 2002.

20. Chang CC, Yang MH, Wen HM, et al. Estimation of total flavonoid content in propolis by two complementary colorimetric methods. J Food Drug Anal. 2002;10(3):178-182.

21. Lee LS, Kim SH, Kim YB, et al. Quantitative analysis of major constituents in green tea with different plucking periods and their antioxidant activity. Molecules. 2014;19(7):9173-9186.

22. Too JC, Kinyanjui T, Wanyoko JK, et al. Effect of sunlight exposure and different withering durations on theanine levels in tea (Camellia sinensis). Food Nutr Sci. 2015;6(11):1014-1021.

23. Yang ZY, Xu Y, Jie GL, et al. Study on the antioxidant activity of tea flowers (Camellia sinensis). Asia Pac J Clin Nutr. 2007;16(Suppl 1):148-152.

24. Lin $\mathrm{YS}, \mathrm{Wu} \mathrm{SS}$, Lin JK. Determination of tea polyphenols and caffeine in tea flowers (Camellia sinensis) and their hydroxyl radical scavenging and nitric oxide suppressing effects. J Agric Food Chem. 2003;51(4):975-978. 
25. Fujimori N, Ashihara H. Biosynthesis of caffeine in flower buds of Camellia sinensis. Ann Bot. 1993;71(3):279-284.

26. Yang Z, Tu Y, Baldermann S, et al. Isolation and identification of compounds from the ethanolic extract of flowers of the tea (Camellia sinensis) plant and their contribution to the antioxidant capacity. LWTFood Sci Technol. 2009;42(8):1439-1443.
27. Chan EWC, Lim YY, Chew YL. Antioxidant activity of Camellia sinensis leaves and tea from a lowland plantation in Malaysia. Food Chem. 2007;102(4):1214-1222.

28. Nibir YM, Sumit AF, Akhand AA, et al. Comparative assessment of total polyphenols, antioxidant and antimicrobial activity of different tea varieties of Bangladesh. Asian Pac J Trop Biomed. 2017;7(4):352-357. 